\title{
Is Justification Necessary for Knowledge?
}

David Sackris and James R. Beebe (University at Buffalo)

Forthcoming in James R. Beebe (ed.), Advances in Experimental Epistemology (Continuum)

Justification has long been considered a necessary condition for knowledge, and theories that deny the necessity of justification have been dismissed as non-starters. In this paper, we challenge this longstanding view by showing that many of the arguments offered in support of it fall short and by providing empirical evidence that individuals are often willing to attribute knowledge when epistemic justification is lacking.

In the early 1990s, Crispin Sartwell $(1991 ; 1992)$ attempted to call into question the traditional view that justification is a necessary condition for knowledge. Unlike some epistemic externalists who suggested that the justification condition be replaced with reliable indication, sensitivity, or some other externalist condition, Sartwell contended that no replacement was necessary. Sartwell's claims were initially met with incredulous stares and were soon largely ignored as their novelty diminished. More recently, other philosophers have taken aim at some of the other purportedly necessary conditions for knowledge. Allan Hazlett (2010; 2012), for example, has pointed to the widespread willingness of individuals to attribute knowledge in the absence of truth, arguing that the ordinary concept of knowledge may not be factive after all. Blake Myers-Schulz and Eric Schwitzgebel (forthcoming) and James Beebe (forthcoming) have gathered empirical data that display folk willingness to attribute knowledge even in the absence of occurrent or dispositional belief. 
In this paper, we seek to reopen the question of whether justification is a necessary condition for knowledge by taking a critical look at some of the philosophical arguments offered in favor of its necessity and by reporting the results of empirical studies that show participants are willing to attribute knowledge when there is insufficient evidence in favor of the belief in question. In Section 1, we revisit Sartwell's reasons for claiming that justification is a criterion for knowledge but not a necessary condition. In Section 2, we respond to objections against Sartwell's view that are offered by Jonathan Kvanvig (2003) and William Lycan (1994). In Section 3, we report the results of empirical tests of some of Sartwell's central claims. We hope that the resulting blend of philosophical argument and empirical results leads philosophers to take more seriously the suggestion that the ordinary concept of knowledge may not include justification.

\section{Sartwell's Argument}

Sartwell begins his attack on the epistemological dogma that knowledge is at least justified true belief by arguing that the obvious importance of having a justification for one's beliefs does not need to be interpreted as showing that justification is a component of knowledge. Rather, he suggests, it might simply be that justification is the most important criterion for knowledge. Asking for justification, after all, is often the best way to determine whether or not someone has

a true belief. Because of the link between epistemic justification and truth, knowing that someone fails to have a good reason for believing a proposition is often what we rely upon most in determining that the belief cannot be trusted. Timothy Williamson (2000) makes an analogous point when he argues that the fact that knowledge entails justification does not show that justification is a constituent of knowledge. 
Unlike Williamson, however, Sartwell also argues that justification is not always required in order to correctly attribute knowledge. He notes that we are often willing to ascribe knowledge in instances of very weak or even absent justification, where, if justification was implicitly part of knowledge, we should otherwise deny that knowledge was present. Sartwell offers the example of a man who correctly believes his son is innocent of a crime in the face of overwhelming evidence against him, basing his belief solely upon the fact that the young man is his son. Sartwell claims that, in practice, we would likely say that he knows his son is innocent, despite the fact that the evidence he possesses does not support an attitude of belief. Sartwell considers several cases along these lines where an agent's belief is eventually vindicated and claims the most natural thing to say is that the agent "knew it all along." In Section 3, we report the results of asking ordinary participants whether the agents in several cases like these had knowledge. In line with Sartwell's predictions, participants were found to be inclined to say the agents 'knew it all along' in contexts where they had no justification or, indeed, where the evidence or justification they possessed pointed to the falsity of their beliefs.

Sartwell $(1991,157-8)$ also considers typical counterexamples offered against his view. Critics often claim that his view implausibly counts as knowledge cases where someone (i) picks a winning horse by closing his eyes and placing his finger at random on a racing form, (ii) dreams that the Pythagorean theorem is true and comes to believe that it is true on that basis, or (iii) forms a true belief on the basis of some delusion. Sartwell argues that in order for these cases to succeed as counterexamples, they need to be examples of true belief but that they are often not plausibly construed as involving belief. Luckily guessing that $p$ does not require believing that $p$. When picking a winning horse at random, you may hope your guess is correct, but you should not believe that it is. In Section 3, we describe the results of presenting three 
'lucky guess' vignettes to participants, the majority of whom judged the agents described therein to lack belief.

In regard to the case of someone forming true beliefs on the basis of dreams or delusions, Sartwell argues that we need to consider what other supporting beliefs the agent possesses and the extent to which the agent fully understands the content of the belief in question. Sartwell $(1991,159)$ contends that if the agent has both a solid understanding of the belief and a genuine belief that it is true (which he claims entails “some degree of serious commitment to the claim”), then it should be counted as an instance of knowledge. As we report in Section 3, the intuitions of ordinary participants are modestly in accord with Sartwell's claims about cases like this.

\section{Objections to Sartwell}

\subsection{Kvanvig's Objections}

Although the main objection against the view that justification is not necessary for knowledge is its alleged counterintuitiveness, some philosophers have offered additional arguments against the view. For example, Kvanvig (2003) believes that Sartwell fails to adequately deflect the challenge posed by some of the counterexamples he considers against his position. When Sartwell asks what we should say about a mental patient who believes that $2+2=4$ on the basis of what she thinks the voices in her head have told her, Sartwell admits that, according to his view, we must ascribe knowledge to her. However, Kvanvig (2003, 6) complains:

[B]ut all we get [from Sartwell] by way of argument for such a denial [of what the common view in philosophy maintains] is a remark that "it is natural in a case such as this one to say that we all know that $2+2=4$; it is 'common knowledge'; in a typical case it would be perverse to ask of any one person how she knows it." None of these 
claims is a sufficient reply to the counterexample, however. It may be natural to say that everyone knows simple arithmetical truths, but it is false. It is natural to say it because the counterexamples are so rare, not because they do not exist.

The problem with Kvanvig's criticism of Sartwell, however, is that Kvanvig fails to consider Sartwell's actual response to the apparent challenge posed by the mental patient. Sartwell (1992, 163) distinguishes two reasons for asking "How do you know?” When we ask this question we may wish to determine if a person really does know the claim in question and does not merely believe it, or we "may be trying to ascertain the believer's overall rationality." That is, we may be trying to determine her overall trustworthiness as an informant, which will affect our further assessment of her claims. If we ask someone how she knows that $2+2=4$, this does not necessarily mean that we are seeking to deny her knowledge. We may instead be trying to ascertain what she considers good grounds. When the mental patient replies that she believes this because the voices in her head told her so, we may determine that her belief is not well-grounded and that she will be a generally unreliable informant without necessarily denying that she has knowledge. In other words, Sartwell thinks we can impugn the mental patient's method of justification without denying that she knows. Kvanvig ignores this component of Sartwell's response to the case. Furthermore, in Section 3 we report the results of a study in which ordinary participants display a willingness to ascribe knowledge to such a mental patient. In addition to asking whether the mental patient knows, we also asked participants if it would be true for the patient to say 'I knew that $2+2=4$ when I was delusional' after she had recovered from her delusion. Individuals were moderately inclined to ascribe knowledge in both instances. 
In spite of the fact that Kvanvig seeks to refute Sartwell's position, much of what Kvanvig goes on to say about the nature of inquiry is actually quite amenable to it. For example, Kvanvig (2003, 54) argues that knowledge is not any more valuable than its parts:

The goal of inquiry, however, is nothing other than getting to the truth and avoiding error, so any property of belief that is valuable from a purely intellectual point of view had better find some connection between that property and truth. So if justification is a valuable property of belief, it cannot be because it has value in and of itself, independently of any relationship to truth.

Sartwell agrees that the goal of inquiry can be specified in terms of true belief without bringing justification into the picture. If our epistemic end is fully achieved when we obtain true belief, Sartwell recommends understanding knowledge as being fully achieved as well. If, as Kvanvig argues, it is not clear how knowledge could be more valuable than true belief and if justification cannot add any value to true belief, perhaps this is a reason for thinking that knowledge simply is true belief.

Kvanvig also criticizes Sartwell's view that a criterion, or means for achieving some goal, cannot also be a constituent of that goal, arguing that this view is patently false. In maintaining that there are clearly some goals where the means to the goal is constitutive of that goal, Kvanvig gives the example of running a successful campaign as something that is both a means to and a constituent of being elected senator. In a second example, he notes that if one has the goal of having a million dollars, acquiring one hundred dollars is both a means to and a necessary constituent of that goal. Pierre Le Morvan (2002, 161-2) offers a similar objection, noting that, for Mill, pleasure is not only a means to but is also constitutive of happiness. We grant that these examples refute Sartwell's unnecessarily strong claims about the relation of 
criteria or means to ultimate goals. However, none of these examples provides any reason for thinking that knowledge is sufficiently like these goals, and neither Le Morvan nor Kvanvig offers any additional reason for thinking that it is.

Consider the fact that an incumbent senator can be re-elected without running a campaign and that a relatively obscure individual who raises his profile in the state as a result of a senate campaign might consider the campaign a success even if he is not elected. Thus, running a successful campaign may be both a means to and a constituent of being elected senator, but it might be neither one. In regard to Kvanvig’s million dollar example, we need to ask whether acquiring knowledge is sufficiently like acquiring a million dollars for us to think that a criterion for knowing must also constitute what it is to know. Kvanvig claims that acquiring one hundred dollars is both a means to and a necessary constituent of acquiring one million dollars. Note that becoming a millionaire is an accumulative goal-the goal is simply an accumulation of its means. However, knowledge does not seem to be a goal of this kind. Knowledge is not simply the accumulation of the means by which it is obtained. It might be correct to say that the more evidence one accumulates for $p$, the closer one comes to knowing that $p$. But even on the traditional epistemological view of knowledge, having an abundance of evidence that $p$ is not the same as knowing that $p$. To the extent that knowledge is disanalogous to the senate campaign and million dollar examples, these examples serve as poor models for what it takes to know something.

\subsection{Lycan’s Objection}

Lycan (1994, 1) begins his critical discussion of Sartwell with the following, understated remarks: 
Crispin Sartwell has recently defended the antiSocratic and outrageous claim that knowledge is, as a matter of philosophical analysis, simply true belief. (Call that claim “TB.”) Sartwell has tried to discredit the obvious presumed counterexamples to TB, and he has also offered an ingenious positive argument in its support. I am unpersuaded by the argument, but in this note I shall merely deduce an ugly consequence from TB taken together with a few harmless assumptions, a consequence I take to be uncontroversially false.

Lycan's focus is more on Sartwell's claim that truth and belief are sufficient for knowledge than upon Sartwell's claim that justification is not necessary. ${ }^{i}$ And although the latter claim is the focus of the present article, we will briefly consider Lycan's objection before moving on. The “ugly” and "uncontroversially false” consequence that Lycan deduces from Sartwell's view is that it could be possible (i) for Sartwell to believe that knowledge is merely true belief, (ii) for Sartwell to believe that he believes that knowledge is merely true belief, and (iii) for both of Sartwell's beliefs to be true. What, you may wonder, is so damning about this possibility? Lycan $(1994,2)$ explains: "it is unlikely that anyone knows any highly controversial philosophical claim to be true, and it is unlikely that Sartwell is so arrogant as to believe he knows [what knowledge is] in particular.”

We find it difficult to believe that the possibility that Sartwell knows what knowledge is counts as an "ugly" and "uncontroversially false" consequence of his view. We would have thought it would have been more damning if his view entailed that it could not be known. Lycan seems not to appreciate the fact (i) that most every philosophical position allows for its own knowability, (ii) that philosophers continually make claims about and hence represent themselves as knowing highly controversial philosophical theses, and thus (iii) that there is nothing special 
about Sartwell in this regard. Furthermore, imagine that one day Sartwell dies and arrives at the pearly gates and that the first question he asks is whether or not he was right about the nature of knowledge. If the answer he receives is 'Yes,' we can easily imagine him saying “I knew it!” and this being a correct thing for him to say. In fact, in Section 3 we report results from a study where we presented participants with a case of this kind—pearly gates included—and found that participants agreed this would be the correct thing to say.

Perhaps the real worry Lycan is trying to pinpoint is not the stated absurdity of taking oneself to know anything in philosophy but rather the fact that Sartwell's view allows knowing anything (philosophical or otherwise) to be far too easy. In order for $S$ to know that $p$, how much epistemic effort is required of $S$ ? Very little, if any. The only thing $S$ needs to do is to believe that p. S's belief also needs to be true, but bringing about the truth of $p$ is (except in exceptional circumstances) not a task that falls to $S$. Rather, that "task" falls to reality. The objection, then, may be that knowledge requires more of a subject than Sartwell's account demands. Sartwell can agree that epistemic life requires effort and that epistemic justification can often be difficult to come by; however, he can contend that this does not show that justification must be part of knowledge. It must also be kept in mind that the thesis of the non-necessity of justification is compatible with justification being required almost all the time. This means that Sartwell need not be interpreted as diminishing the important role that epistemic justification plays in our epistemic lives. Relatedly, Sartwell can agree that the norms of assertion license an assertion of $p$ or a claim to know $p$ only when one has a sufficient amount of justification for these claims. This means that on Sartwell's view someone could know $p$ and yet not be justified in asserting that $p$. Given the murkiness surrounding the issue of norms of assertion, however, it is far from clear that this should count as an objection against his view. 


\section{Empirical Studies}

As we noted at several points above, many of Sartwell's key claims about the intuitively correct verdict concerning potential counterexamples to his position are eminently testable and that we have indeed tested them. In the present section, we report the results of our studies.

One of the most common counterexamples offered against the thesis of the non-necessity of justification is a case where someone picks a winning horse by closing his eyes and placing his finger at random on a racing form. Because Sartwell (1991, 157-8) claimed that cases like this are ones where it does not seem that a belief is present, we asked experimental participants whether or not the protagonists in the following three vignettes had beliefs about the relevant propositions:

RACETRACK: Jack decides to spend the day at the race track with his friends, although he does not know much about horses. He merely wishes to have a good time and hopefully to win a little money. In order to decide which horse to bet on, Jack simply closes his eyes and places his finger on the racing form. Whichever horse his finger lands on, he then places the minimum bet on that horse. This time, Jack's finger lands on the horse named 'Buy A Nose.' Jack then dutifully places the minimum bet on Buy A Nose and moves towards the race track to observe the upcoming race. To his delight, Buy A Nose ends up winning the race.

Q1: Please indicate the extent to which you agree or disagree with the following claim: “At the time when Jack placed his bet, he believed that Buy A Nose would win the race." BASKETBALL: Susan doesn’t know anything about college basketball but decides to fill out a college basketball bracket in order to participate in a competition being held at her 
office. ${ }^{\text {ii }}$ She makes predictions completely at random about which teams will defeat other teams in order to fill out the bracket, not knowing anything about the teams or even where most of them are located. She then dutifully turns in her bracket to compete in the office competition. To her delight, Susan ends up winning the competition.

Q1: Please indicate the extent to which you agree or disagree with the following claim: "At the time when Susan turned in her bracket, she believed that she would win the office competition.”

ACADEMY AWARDS: Mike doesn't know anything about the nominees for this year's Academy Awards, but he decides to fill out a questionnaire that asks him to predict who will win each of the prizes in order to participate in a competition being held at his office. Mike makes predictions completely at random about which stars will win using a list of nominated actors and actresses he was given. He then dutifully turns in his questionnaire to compete in the office competition. To his delight, Mike ends up winning the competition.

Q1: Please indicate the extent to which you agree or disagree with the following claim: "At the time when Mike turned in his questionnaire, he believed that he would win the office competition.”

In each of the studies described in this section, when participants were asked to indicate agreement or disagreement with a belief or knowledge ascription, they reported their answers using a seven-point scale marked with the labels 'Completely Disagree,' 'Mostly Disagree,' 'Slightly Disagree,' 'Neither Agree nor Disagree,' 'Slightly Agree,' 'Mostly Agree,' and ‘Completely Agree.’ In a between-subjects design, 98 undergraduate students (average age = 22, 64\% female, 74\% Anglo-American) from the northeastern United States completed online 
questionnaires hosted at vovici.com in exchange for extra credit in an introductory course. Results are represented in Figure 1.

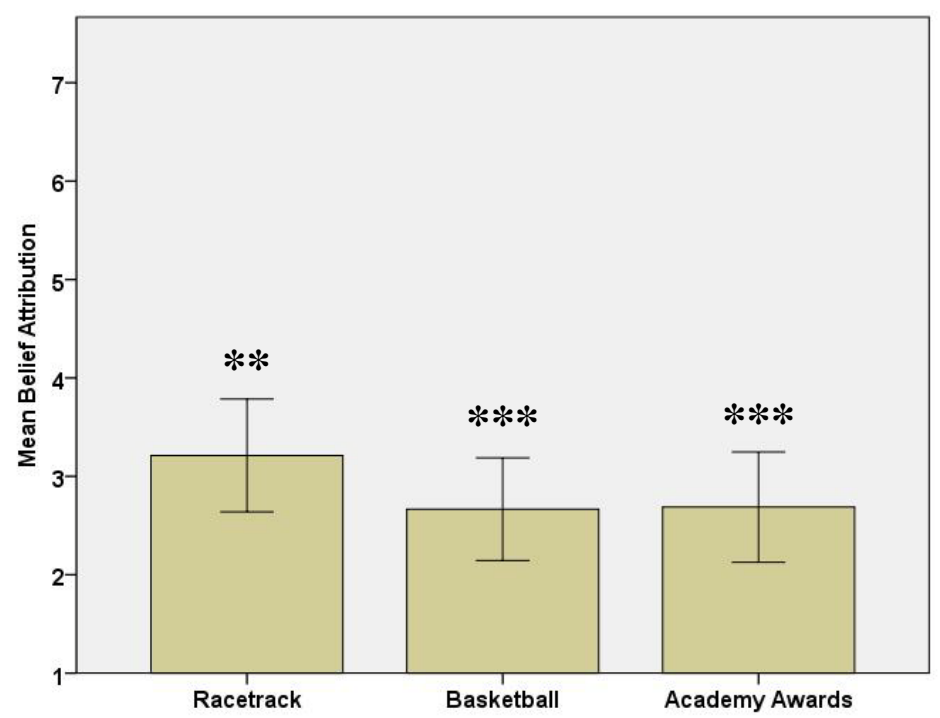

Figure 1. Mean belief attributions in the Racetrack (3.21), Basketball (2.67), and Academy Awards (2.69) conditions. An '*,' ‘**, or '***' indicates that the mean differs significantly from the neutral midpoint at either the .05, the .01 , or the .001 level. Error bars represent 95\% confidence intervals in all figures.

As Sartwell predicted, participants displayed a disinclination to attribute belief in these cases. Averaging across all three cases, $62.2 \%$ of participants gave answers that fell below the neutral midpoint. ${ }^{\text {iii }}$ The foregoing cases are brought forward as examples where truth and belief are supposed to be present without justification. The intuitively correct thing to say about them is that the protagonists do not have knowledge about the relevant propositions ${ }^{\text {iv }}$, and this is supposed to cast doubt upon the thesis of the non-necessity of justification. However, as we can see, they are not taken to be instances of belief at all. Consequently, they fail to serve as effective counterexamples to the non-necessity thesis.

A second class of purported counterexamples to the non-necessity of justification thesis 
concerns cases where a true belief is obtained in an epistemically unworthy manner as the result of cognitive malfunction or some other improper grounding. Each of the following vignettes is based upon examples discussed by Sartwell:

CLINTON1: Sunil is an exchange student who has recently become highly delusional. He claims that demons are talking to him inside his head and that they tell him all sorts of things. Sunil believes everything the demons tell him. One of the things the demons tell him is that Hillary Clinton is the current U.S. Secretary of State. Sunil has never followed American politics very closely, but he comes to believe that Hillary Clinton is Secretary of State on this basis. It turns out, of course, Hillary Clinton really is the current U.S. Secretary of State.

Q1: Please indicate the extent to which you agree or disagree with the following claim: "Sunil knows that Hillary Clinton is the current U.S. Secretary of State."

CLINTON2: Sunil is an exchange student who has recently become highly delusional. He claims that demons are talking to him inside his head and that they tell him all sorts of things. Sunil believes everything the demons tell him. One of the things the demons tell him is that Hillary Clinton is the current U.S. Secretary of State. Sunil has never followed American politics very closely, but he comes to believe that Hillary Clinton is Secretary of State on this basis. It turns out, of course, Hillary Clinton really is the U.S. Secretary of State. After Sunil eventually recovers from his state of delusion, he begins to learn about American politics. While reading about Hillary Clinton's current role as Secretary of State, he thinks to himself "I first acquired knowledge of this fact back when I was delusional.” 
Q1: Please indicate the extent to which you agree or disagree with the following claim: "When Sunil was delusional, he knew that Hillary Clinton was the current U.S. Secretary of State.”

SQUARE ROOT1: Jordan, a college aged student, has become highly delusional. He claims that demons are talking to him inside his head and that they tell him all sorts of things. Jordan believes everything the demons tell him. One of the things that the demons tell him is that 125 is the square root of 15,625 , and he comes to believe that 125 is the square root of 15,625 on this basis. It turns out that 125 really is the square root of 15,625 .

Q1: Please indicate the extent to which you agree or disagree with the following claim: "Jordan knows that 125 is the square root of 15,625."

SQUARE ROOT2: Jordan, a college aged student, has become highly delusional. He claims that demons are talking to him inside his head and that they tell him all sorts of things. Jordan believes everything the demons tell him. One of the things that the demons tell him is that 125 is the square root of 15,625 , and he comes to believe that 125 is the square root of 15,625 on this basis. After Jordan eventually recovers from his state of delusion, he begins to work on some math problems. Using a calculator, he finds that the square root of 15,625 is 125. Jordan then thinks to himself "I first acquired knowledge of this fact back when I was delusional.”

Q1: Please indicate the extent to which you agree or disagree with the following claim: "When Jordan was delusional, he knew that 125 was the square root of 15,625." THEOREM: Brian is a 10 year old boy who has just begun to study geometry. One night he goes to sleep and dreams that the square of the hypotenuse of a right triangle is equal 
to the sum of the squares of its other two sides. On the basis of this dream, he comes to believe the Pythagorean Theorem. A few days later in school his teacher introduces the Pythagorean Theorem for the first time in class. Brian thinks to himself "I already knew that the square of the hypotenuse of a right triangle is equal to the sum of the squares of its other two sides.”

Q1: Please indicate the extent to which you agree or disagree with the following claim: "Brian already knew that the Pythagorean Theorem was true."

In the first four cases above, the protagonist experiences a psychotic episode in which he hears voices telling him either that a traditionally $a$ priori proposition is true or that a traditionally $a$ posteriori proposition is true. In each case, the protagonist believes what the voices say, and the belief turns out to be correct. In Clinton1 and Square Root1, we had participants consider the protagonists' beliefs while they were still suffering from their delusions, whereas in Clinton2 and Square Root2 we portrayed the protagonists after they had recovered and were reflecting back upon their delusional state. In the fifth case, we had the protagonist form a belief on the basis of a dreaming episode, which-like hearing voices in one's head-is widely taken to be an epistemically inappropriate basis for belief.

In a between-subjects design, 189 undergraduate students (average age $=21,64 \%$ female, 75\% Anglo-American) from the northeastern United States completed online questionnaires hosted at vovici.com in exchange for extra credit in an introductory course. Results are depicted in Figure 2. 


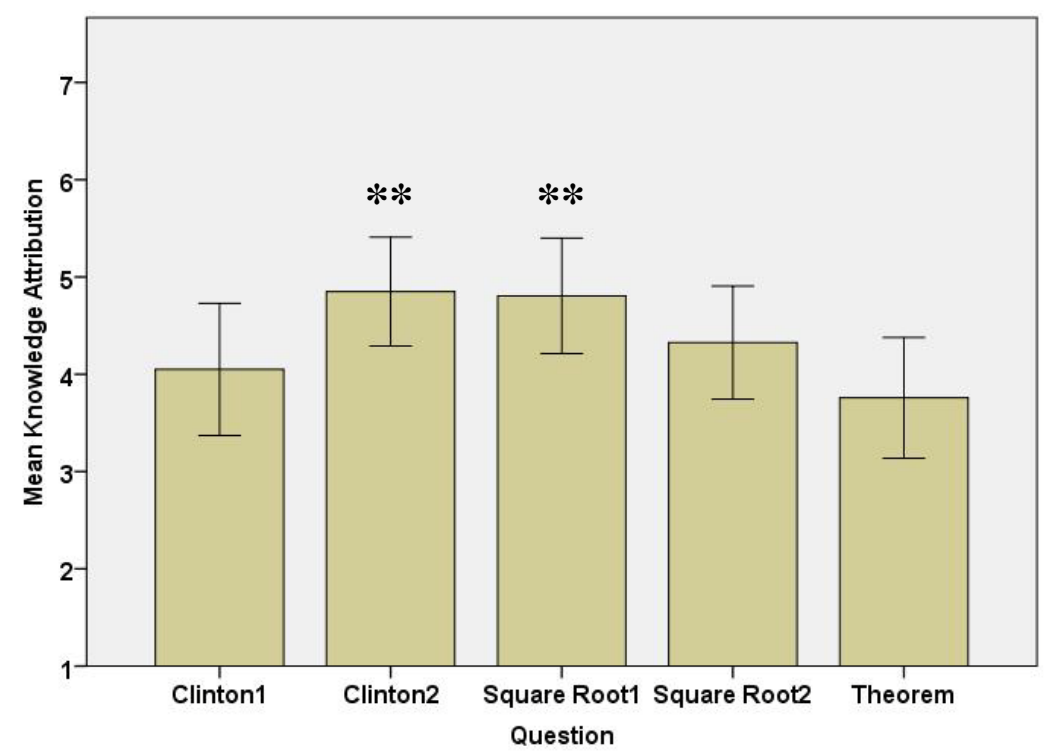

Figure 2. Mean knowledge attributions in the Clinton1 (4.05), Clinton2 (4.85), Square Root1 (4.81), Square Root2 (4.33), and Theorem (3.76) conditions.

In two of the conditions (Clinton2 and Square Root1) participants' mean knowledge attributions fell significantly above the neutral midpoint. ${ }^{\mathrm{v}}$ However, averaging across all cases, only 34.3\% of participants gave responses that fell below the midpoint, whereas $54.5 \%$ gave answers above the midpoint. If the ordinary concept of knowledge requires that beliefs be epistemically wellfounded, it seems that a sizable portion of philosophically untrained individuals are handling the concept rather poorly.

The third and final set of cases we tested involved protagonists whose evidence went against their beliefs but who had true beliefs nonetheless. In a between-subjects design, 352 participants (average age $=28,61 \%$ female, 77\% Anglo-American) from the United States were presented with one of the following vignettes and one of the two questions that appears after each vignette:

JOHN: John's daughter has been accused of murder. Even though she lacks a strong alibi and the police have compelling evidence against her, John feels she must be innocent. 
After several very stressful weeks, the actual murderer finally comes forward and confesses.

Q1: Please indicate the extent to which you agree or disagree with the following claim: "John knew all along that his daughter was innocent."

Q2: In light of the information available to John BEFORE the actual murderer came forward and confessed, how likely was it that John's daughter was innocent?

SANDRA: The team of doctors responsible for treating Sandra's cancer told Sandra's husband, Mickey, that there was virtually no chance she would be able to beat the cancer and survive for more than a few months. In spite of what the doctors told him, Mickey was convinced that she would beat the cancer. In the end, Mickey's wife survived the cancer and remained cancer free for more than 35 years.

Q1: Please indicate the extent to which you agree or disagree with the following claim: "Mickey knew all along that his wife would survive the cancer."

Q2: In light of the information available to Mickey BEFORE Sandra survived the cancer and remained cancer free for 35 years, how likely was it that Sandra would survive the cancer?

BOB1: Bob is a scientist who has devoted his entire career to defending the view that prolonged cell phone use causes brain tumors. No other scientist, however, has accepted Bob’s theory. In fact, his papers are continually rejected for publication, and funding organizations always reject his requests for grant money. One day Bob dies and arrives at the entrance to heaven. The first question Bob asks upon arrival in heaven is whether or not he was right about the relationship between cell phone use and brain tumors. He 
learns that his widely disparaged theory is correct. Bob exclaims "I knew that prolonged cell phone use caused brain tumors!”

Q1: Please indicate the extent to which you agree or disagree with the following claim: "Bob knew all along that prolonged cell phone use caused brain tumors.”

Q2: In light of the information available to Bob BEFORE Bob dies and goes to heaven, how likely was it that Bob’s theory was correct?

Thus, we asked some participants whether the protagonists had knowledge, and we probed other participants about the strength of the protagonists' evidence. We also employed a second version of the scenario involving Bob (viz., Bob2), where the statement 'He learns that his widely disparaged theory is correct' was followed by 'even though the experiments he tried to use to prove his theory were flawed.' This was added to make the evidence against Bob’s belief even stronger than in Bob1. The same questions that appear after Bob1 were used with Bob2.

Participants responded to each of the Q1 questions using the same seven-point scale used in the above experiments (ranging from 'Completely Disagree' to 'Completely Agree'). Participants responded to the Q2 questions using a seven-point scale that was labeled with 'Highly Unlikely,' 'Moderately Unlikely,' 'Somewhat Unlikely,' 'Neither Likely nor Unlikely,' 'Somewhat Likely,' 'Moderately Likely,' and 'Highly Likely.' Results are depicted in Figure 3. 


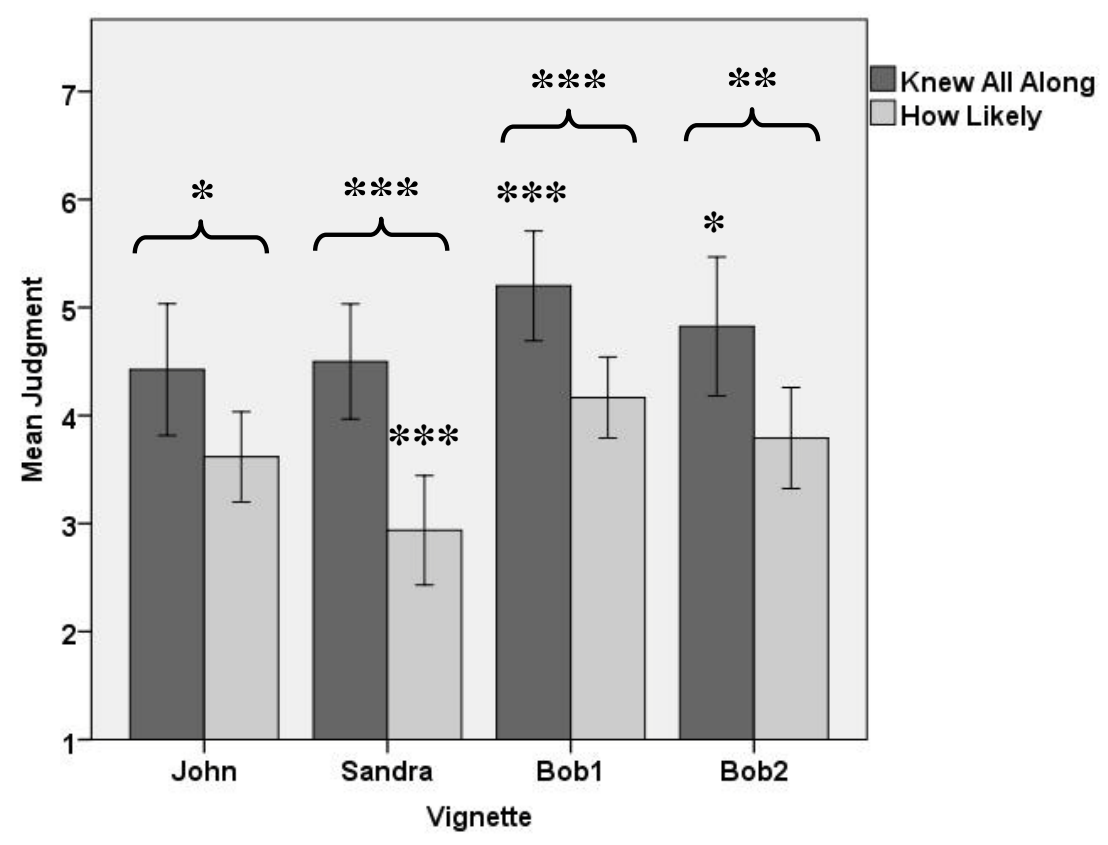

Figure 3. Mean knowledge attributions and likelihood ratings in the John (4.43, 3.62), Sandra (4.50, 2.94), Bob1 (5.20, 4.17), and Bob2 (4.82, 3.79) conditions. An '*,' '**,' or '***' with a bracket indicates a statistically significant difference between pairs of conditions at either the .05 , the .01 , or the .001 level.

Mean knowledge attributions in the two Bob conditions fell significantly above the neutral midpoint, while the mean likelihood rating in the Sandra case fell significantly below the midpoint. ${ }^{\text {vi }}$ A set of independent samples t-tests confirms that each of the four mean knowledge ratings differs significantly from its associated mean likelihood rating. ${ }^{\text {vii }}$ Thus, participants were more inclined to attribute knowledge to the protagonists in these four cases than they were to attribute evidence that made the protagonists’ beliefs more likely to be true than not.

Although the scale used for rating the extent of participants’ agreement or disagreement with a knowledge attribution employed different verbal anchors than the scale used for rating their assessments of how likely certain outcomes were, we believe that a comparison between the two sets of participant responses is instructive. Both scales, for example, included a neutral midpoint, with deviations in two directions from this point. Because participants were 
significantly more inclined to think that the protagonists in the John, Sandra, Bob1, and Bob2 vignettes have knowledge than they were to think that the protagonists had decent evidence for their beliefs (where evidence is construed in accord with philosophical tradition as having a probabilistic connection to the truth), we contend that these results tell against the necessity of justification thesis—at least to the extent that it purports to model folk knowledge attributions.

\section{Conclusion}

It is important to distinguish the following two claims:

(1) Justification is not a necessary condition for knowledge.

(2) Knowledge is merely true belief.

The second claim is stronger than the first in several respects. For our purposes, the most important is that (2) implies that justification is never required for knowledge. If truth and belief are present, knowledge is guaranteed to be so as well. By contrast, however, (1) is compatible with justification being necessary some of the time or even most of the time. It simply denies that justification is always required for knowledge. This means that a single, compelling counterexample in which an agent's unjustified true belief fails to seem like a case of knowledge would do damage only to the second claim. Whereas Sartwell defends both claims in his writings, our main goal has been to reconsider the case against the former.

We do not think there is any simple and direct argument from 'Many philosophically untrained individuals are willing to attribute knowledge in the absence of solid evidence or justification' to the truth of (1). Consequently, we do not take our data to have established (1). However, we contend that our results undermine arguments against (1) that are based upon 
armchair appeals to what are assumed to be widely shared intuitions about the necessity of justification.

According to our interpretation of the history of appeals to epistemic intuitions, (i) epistemologists used to appeal to the intuitions of both philosophical experts and the philosophically untrained in order to support their favored accounts of knowledge, until (ii) experimental philosophers came along and showed that the intuitions of the masses were often surprisingly different from what had been expected, after which time (iii) epistemologists claimed they had never been interested in folk intuitions in the first place. We think that one of the great benefits of experimental philosophy has been the motivation it has provided for philosophers to consider what (if anything) philosophical expertise consists in and how it can be detected and measured. Our results leave open the possibility that (1) is false, and that the epistemic intuitions of those with genuine philosophical expertise would support this fact. However, the vast majority of epistemologists who both maintain that (1) is false and reject the reliance upon folk intuitions in philosophical theory formation want to fashion theories of our ordinary, shared concept of knowledge. To the extent that they take themselves to be analyzing a concept possessed by the average person on the street and not a technical notion known only to specialists, our results provide a challenge to the longstanding view that justification is necessary for knowledge.

We believe that the folk conception of knowledge is more contextually variable and multi-faceted than most philosophical accounts of knowledge have assumed. We hope that the present set of arguments and studies contributes to a better understanding of its richness and complexity. 


\section{References}

Beebe, J. R. (forthcoming), 'A Knobe effect for belief ascriptions'. The Review of Philosophy and Psychology.

Hazlett, A. (2010), 'The myth of factive verbs'. Philosophy and Phenomenological Research 80, 497-522.

Hazlett, A. (2012), 'Factive presupposition and the truth condition on knowledge'. Acta Analytica, 27, 461-78.

Kvanvig, J. (2003), The Value of Knowledge and the Pursuit of Understanding. New York: Cambridge University Press.

Le Morvan, P. (2002), ‘Is mere true belief knowledge?’ Erkenntnis , 56, 151-68.

Lycan, W. (1994), ‘Sartwell’s minimalist analysis of knowing’. Philosophical Studies, 73, 1-3.

Myers-Schulz, B. and Schwitzgebel, E. (forthcoming), 'Knowing that p without believing that p’. Noûs.

Sartwell, C. (1991), 'Knowledge is merely true belief’. American Philosophical Quarterly, 28, 157-65.

Sartwell, C. (1992), 'Why knowledge is merely true belief'. Journal of Philosophy , 89, 167-80.

Williamson, T. (2000), Knowledge and Its Limits. New York: Oxford University Press.

${ }^{\mathrm{i}}$ Cf. Sec. 4 for further discussion of this distinction and the difference it makes to the present article.

ii In the United States, 'filling out a bracket' means predicting which teams will win which matches in a tournament.

iii One-sample t-tests revealed that each mean fell significantly below the neutral midpoint. Racetrack: $t(32)=-2.802, p<.01, r=.44$ (medium effect size). Basketball: $t(32)=-$ 
5.204, $p<.001, r=.68$ (large effect size). Academy Awards: $t(31)=-4.777, p<.001, r=.65$ (large effect size)

${ }^{\text {iv }}$ Using an independent set of participants, we confirmed this common supposition. The mean knowledge attributions in the three cases were near the floor: 1.35, 1.43, and 1.48, respectively (on a scale from 1 to 7 ).

${ }^{\mathrm{v}}$ Clinton1: $t(39)=.149, p>.05$. Clinton2: $t(32)=3.076, p<.01, r=.48$ (medium effect size). Square Root1: $t(35)=2.756, p<.01, r=.42$ (medium effect size). Square Root2: $t(39)=$ 1.131, $p>.05$. Theorem: $t(32)=-.796, p>.05$.

vi John Knew All Along: $t(39)=1.410, p>$.05. John How Likely: $t(46)=-1.845, p=$ .071. Sandra Knew All Along: $t(39)=1.900, p=.065$. Sandra How Likely: $t(47)=-4.223, p<$ $.001, r=.52$ (large effect size). Bob1 Knew All Along: $t(39)=4.778, p<.001, r=.61$ (large effect size). Bob1 How Likely: $t(47)=.893, p>.05$. Bob2 Knew All Along: $t(39)=2.594, p<$ $.05, r=.38$ (medium effect size). Bob2 How Likely: $t(47)=-.896, p>.05$.

vii John: $t(85)=2.259, p<.05, r=.24$ (small effect size). Sandra: $t(86)=4.274, p<.001$, $r=.42$ (medium effect size). Bob1: $t(86)=3.364, p<.001, r=.34$ (medium effect size). Bob2: $t(86)=2.676, p<.01, r=.28$ (small effect size). 